$\mathrm{AB} 1314$

ROLE OF NEUTROPHIL TO LYMPHOCYTE RATIO, MONOCYTE TO LYMPHOCYTE RATIO, PLATELET TO LYMPHOCYTE RATIO, EOSINOPHIL TO LYMPHOCYTE AND BASOPHILE TO LYMPHOCYTE RATIO IN ASSESSING DISEASE ACTIVITY IN SPONDYLOARTHRITIS

Salomé Garcia, Bruno Miguel Fernandes, Sara Ganhão, Raquel Ferreira, Miguel Bernardes, Georgina Terroso, Lúcia Costa. Centro Hospitalar Universitário São João, Rheumatology, Porto, Portugal

Background: Neutrophil to lymphocyte ratio (NLR), monocyte to lymphocyte ratio (MLR), platelet to lymphocyte ratio (PLR), eosinophil to lymphocyte ratio (ELR) and basophile to lymphocyte ratio (BLR) have been demonstrated to be promising systemic inflammation markers. NLR, MLR and PLR have been associated with disease activity in Spondyloarthritis $(\mathrm{SpA})$ but the results remain conflicting.

Objectives: We aim to determine the role of NLR, MLR, PLR, ELR and BLR in assessing disease activity in SpA.

Methods: Observational retrospective study was performed including consecutive patients with the diagnosis of SpA (according to ASAS classification criteria) followed at our Rheumatology Department. Demographic, clinical (including BASDAI, BASFI, ASDAS ESR and ASDAS CRP indices) and laboratorial data were collected from our national database at baseline and 6 months after initiation of a tumour necrosis factor inhibitor (TNFi). Correlations between variables were studied using Spearman correlation analysis and comparison between groups was performed using Wilcoxon test.

Results: The mean age of patients $(n=297)$ was 41 years old ( \pm 12 ), $160(53.9 \%)$ were males with median disease duration of 12.4 (IQR 14.8) years. Two hundred and seven patients (69.7\%) had Ankylosing Spondylitis, $26(8.8 \%)$ Inflammatory Bowel Disease related SPA and 36 (12.1\%) Undifferentiated SpA. Seventy-three (24.7\%) patients were taking glucocorticoids and regarding conventional synthetic disease-modifying antirheumatic drugs (csDMARDs) use before starting the TNFi: 188 (63.3\%) were not under any CsDMARD and the remaining ones were under Sulfasalazine $(70,23.6 \%)$, Methotrexate (MTX) (21, 7.1\%), Azathioprine (AZA) (5, 1,7\%), Leflunomide $(1,0.3 \%)$ or associations between Sulfasalazine and AZA or MTX. Regarding the iTNF the majority of patients initiate Adalimumab $(n=168,28.6 \%)$, Golimumab $(n=61,25.6 \%)$ or Infliximab $(n=57,23.9 \%)$. The majority of patients had very high or high disease activity at baseline $(59.6 \%$ and $31.3 \%$, respectively); mean ASDAS CRP was $3.85( \pm 0.99)$, mean ESR was $30.1 \mathrm{~mm} / \mathrm{h}( \pm 21.9)$ and mean CRP was $36.9 \mathrm{mg} / \mathrm{L}( \pm 113.9)$. The post-treatment mean ESR, CRP, ASDAS-CRP, ASDAS-ESR and BASDAI were significantly lower than mean baseline values, as they were also for NLR, MLR and PLR $(p<0.01)$

At the baseline evaluation, in anti-TNF naive patients, NLR and MLR were positively correlated with the majority of parameters evaluated: ESR level $(r=0.322 ; \quad r=0.203, \quad p<0.01$ respectively $), \quad$ CRP level $(r=0.475$; $r=0.221, \quad p<0.01$ respectively), ASDAS-CRP $(r=0.255 ; \quad r=0.192, \quad p<0.01$ respectively), ASDAS-ESR $(r=0.257 ; \quad r=0.206, \quad p<0.01$ respectively $)$ and BASMI ( $r=0.288 ; r=0.150, p<0.01$ respectively). No correlations were found with BASDAI. PLR was positively correlated with ESR level $(r=0.379$, $p<0.01)$, CRP level $(r=0.331, p<0.01)$, ASDAS-CRP $(0.215, p<0.01)$ and ASDAS-ESR $(r=0.208, p<0.01)$. No correlations were found between those parameters and ELR or BLR.

At the evaluation 6 months after introducing a TNFi, we found less and weaker correlations than in naïve patients: NLR and PLR correlate positively with CRP $(r=0.302 ; 0.315, p<0.01$ respectively) and, reaching lower statistical significance, PLR correlate also with ASDAS-ESR (0.156, $\mathrm{p}<0.05)$; NLR with BASMI $(0.184, p<0.05)$ and ESR $(0.179, p<0.05)$; MLR with CRP $(0.173, p<0.05)$.

Conclusion: NLR, MLR and PLR may reflect disease activity and could represent future inexpensive potential parameters to evaluate disease activity or severity in $\mathrm{SpA}$.

Disclosure of Interests: None declared

DOI: 10.1136/annrheumdis-2019-eular.2243

\section{AB1315 MIOSITIS AUTOANTIBODIES PROFILE: DIAGNOSTIC RELEVANCE}

Alicia García Dorta ${ }^{1}$, Yvelise Barrios ${ }^{2}$, Sonia Peña Montelongo ${ }^{1}$, Hernández Díaz Marta', Andrea Monroy Calero', Beatriz Rodríguez Lozano ${ }^{1}$, Andrés Franco ${ }^{2}$, Sagrario Bustabad $1 .{ }^{1}$ Hospital Universitario de Canarias, Reumatology, La Laguna, Spain; ${ }^{2}$ Hospital Universitario de Canarias, Inmunology, La Laguna, Spain

Background The idiopathic inflammatory myopathies (IIM) are autoimmune systemic diseases that can affect several organs, but with special impact in the muscular-skeletal system. Autoimmunity is believed to have a role in its pathogenesis, with the presence of Autoantibodies (Abs) in more than $50 \%$ of the patients. Those Abs have been classified into Myositis-Specific Autoantibodies (MSA), with the Anti-Jo being the most frequent1-2; and Myositis-Associated autoantibodies (MAA), also found in other connective tissue diseases. The availability of a multiplex analysis (Myositis Profile) has opened up new possibilities for the specific investigations of these Abs in the day-to-day clinical practice 1-2.

Objectives To describe the clinical spectrum and the Ab results obtained from the myositis profile in patients (hospital admissions and ambulatory) of a tertiary university hospital.

Methods Retrospective descriptive study (January to June 2018) of the results obtained on the myositis profile. The clinical characteristics of the sample is described in the context of the Abs results. We considered that applications of this Myostis Ab Profile were justified if they had EPID and/or if they had 2 or more clinical and/or analytical characteristics associated with MII. Myositis Profiles consisted in a solid phase ELISA from EuroLINE MII 16 Antigens (Mi2-alfa, Mi2-beta, TIF1gamma, MDA5, NXP2, SAE1, Ku, Pm-Scl100, Pm-Scl75, Jo-1, SRP, PL7, PL12, EJ, OJ, RO-52). Statistical analysis SPSS 17.0.

Results A total of 165 applications were studied, $47 \%$ requested by Reumatology, $30 \%$ by Neumology and $9 \%$ by Internal Medicine. Justifications were: ILD ( $n=49)$; osteomuscular $(n=30)$, connective diseases $(n=29)$, myositis $(n=17)$, CKNAC elevation $(n=11)$, fibromyalgia $(n=7)$ and others $(n=22) .41 \%$ men and $59 \%$ women, Average Age 58 (+/- 15 years). We observe an increase of CKNAC, GOT, GPT y LDH in $21 \%, 12 \%, 19 \%$ y $24 \%$ respectively. They refer muscle weak ness in $18 \%$, Raynaud's phenomenon in $10 \%$, arthritis in $15 \%$, heliotrope eruption in $4 \%$, Gottrons's papules in $1 \%$ and mechanics hand in $1 \%$. Using the criteria specified in the method section, $52 \%$ of the applications were justified, being the $57 \%$ of them as ILD study. $32(20 \%)$ of the 165 profiles were positive. 10 of the 49 patients with ILD had positive Abs (figure 1), $90 \%$ me. 8 of the 17 patients with myositis had positive Abs (figure 1), $63 \%$ women. $75 \%$ of them (6/8) were positive for MSA, the $65 \%$ were Anti-Jo1 (4/6). 1 patient with myositis associated with neoplasia (anti-OJ). 2 of 3 muscle biopsy were consistent with MII. 2 of 7 EMG were compatible. The 14 remaining patients had some positive Abs (figure 2): 7 connective tissue diseases studies, 4 osteomuscular pathology, 1 auto-inflammatory disease, 1 CK-NAC elevation and 1 with inflammatory bowel disease (IBD).
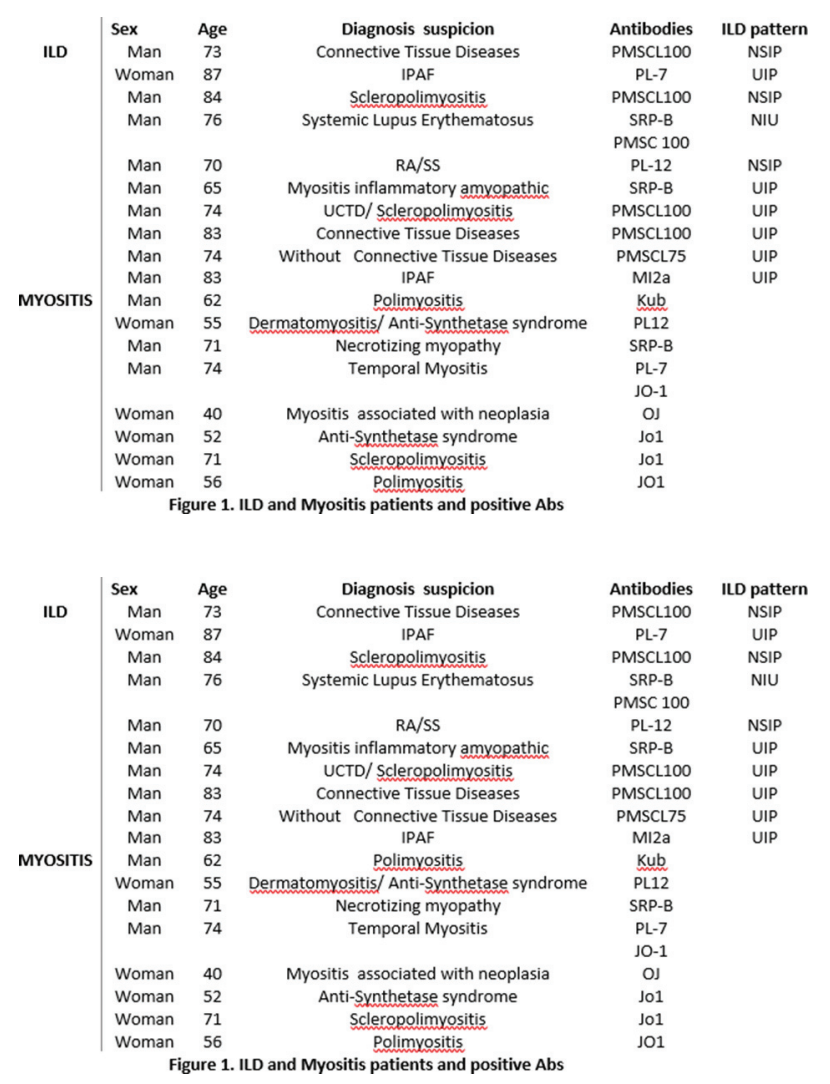

Conclusion The use of this Ab profile as a protocol for patients with ILD without myositis clinical features, allowed us to classify a group of patients with autoimmune diseases. In this group, the distribution by sex, 9 males and 1 woman is highlighted. We achieved the diagnosis on the $47 \%$ of patient with myositis clinical suspicion. The usefulness of myositis profile allowed us to detect antibodies which are not in other immunologic assays. Distribution by sex, 3 men and 5 women. 